Research Article

\title{
Free Vibrations with Large Amplitude of Axially Loaded Beams on an Elastic Foundation Using the Adomian Modified Decomposition Method
}

\author{
Desmond Adair $\mathbb{D}^{\mathrm{D}},{ }^{1}$ Askar Ibrayev ${ }^{\mathrm{D}},{ }^{2}$ Alima Tazabekova ${ }^{\mathrm{D}},{ }^{1}$ and Jong R. Kim ${ }^{2}$ \\ ${ }^{1}$ Department of Mechanical \& Aerospace Engineering, Nazarbayev University, Astana 010000, Kazakhstan \\ ${ }^{2}$ Department of Civil \& Environment Engineering, Nazarbayev University, Astana 010000, Kazakhstan \\ Correspondence should be addressed to Desmond Adair; dadair@nu.edu.kz
}

Received 12 June 2018; Accepted 27 January 2019; Published 26 February 2019

Academic Editor: Francesco Pellicano

Copyright (c) 2019 Desmond Adair et al. This is an open access article distributed under the Creative Commons Attribution License, which permits unrestricted use, distribution, and reproduction in any medium, provided the original work is properly cited.

\begin{abstract}
Analytical solutions describing free transverse vibrations with large amplitude of axially loaded Euler-Bernoulli beams for various end restrains resting on a Winkler one-parameter foundation are obtained using the Adomian modified decomposition method (AMDM). The AMDM allows the governing equation to become a recursive algebraic equation, and, after some additional simple mathematical operations, the equations can be cast as an eigenvector problem whose solution results in the calculation of natural frequencies and corresponding closed-form series solution of the mode shapes. Important to the use of the Adomian modified decomposition method is the treatment of the nonlinear Fredholm integral coefficient, which forms part of the governing equation. In addition to the calculation of natural frequencies and mode shapes, investigations are made of the effects on the free vibrations of the Winkler parameter and of increasing the axial loading.
\end{abstract}

\section{Introduction}

Uniform slender beams resting on an elastic foundation, while subjected to axial loading, are common in structural systems undergoing actual operating conditions. Analysis of such systems, both linear and nonlinear, has been of interest to civil and railway engineering. For example, when ambient temperatures increase, rails and concrete slabs, often used in urban transport systems, tend to expand, so causing compressive in-plane forces, leading to changes in natural frequencies and eventually to buckling. In-plane compressive forces are also found in prestressed beams. If the amplitudes of the vibrations remain small, the governing equation is usually in the form of a linear differential equation which is relatively simple to solve. However for large amplitude vibration, nonlinear terms are introduced into the governing equation which needs to be treated. Rails and concrete slabs often rest on foundations generally classified as elastic, viscoelastic, Winkler, and Pasternak.
Boundary value problems (BVPs) have been the subject of several analytical methods, recently developed, to calculate beams with relatively simple configurations. The variational iteration method (VIM) often attributed to $\mathrm{He}$ [1] is a modification of a general Lagrange multiplier method and has been used as a powerful tool for solving ordinary differential equations $[2,3]$. Another recent method developed is the homotopy perturbation method (HPM) $[4,5]$ which has been used for problems involving nonlinear differential equations. Less recent methods used to investigate the vibration problem for nonuniform EulerBernoulli beams have been the Rayleigh-Ritz method [6], closed-form solutions [7], and Green's function method [8]. Several methods have used the Frobenius series [9] and also by discretizing the beam into beam elements [10]. There have been some early studies of vibrating beams under axial loading [11-13], where the effect of increasing the axial loading on the mode shapes and natural frequencies of the beam was investigated. There has been some work already 
done for large amplitude vibration. Bhashyam and Prathap [14] used the Galerkin finite element method to study nonlinear vibration, and Özkaya [15] calculated the response of a beam mass system with clamped ends by applying a method known as the method of multiple scales.

For beams resting on foundations, an understanding of the beam-foundation interaction is needed. The foundation increases resistance to movement and can significantly change the modal characteristics of the beam. Many practical cases in engineering related to foundation-beam interaction can be modelled by assuming the beam resting on an elastic foundation with the Winkler elastic foundation model [16] used extensively. This model assumes the foundation to be made up of an infinitely many closedspaced linear springs and is a one-parameter model. A limitation of this model is the assumption that there is no interaction between the springs. To overcome this defect, several two-parameter models have been suggested, such as Filonenko-Borodich, Pasternak, and Vlasov and Leontiev foundation models [17]. Studies using a constant Winkler foundation can be found in the literature [18-20].

In the present work, the adomian modified decomposition method [21, 22] is utilized to calculate free transverse vibration characteristics of axially loaded Euler-Bernoulli beams with various end restrains, resting on a Winkler one-parameter foundation. The method is chosen as it has proved efficient and accurate $[23,24]$ for solving linear and nonlinear differential equations, and it has the advantage of computational simplicity. In addition, it does not involve linearization, discretization, perturbation, or $a$ priori assumptions, which may alter the physics of the problem considered [21]. For the AMDM, the solution is considered to be the sum of an infinite series with rapid convergence [25]. Using the AMDM, the governing differential equation becomes a recursive algebraic equation and the boundary conditions become simple algebraic frequency equations, which are suitable for symbolic computation. After some simple algebraic operations on the frequency equations for any $i^{\text {th }}$ natural frequency, the closed-form series solution of any $i^{\text {th }}$ mode shape can be obtained. Calculations are made for clamped-free and clamped-clamped boundary conditions together with an investigation of the effects of increasing the axial loading and Winkler parameter on the natural vibrations.

\section{Principle of Adomian Modified Decomposition Method (AMDM)}

The basic theory of AMDM is briefly stated here. Consider the equation

$$
F y(x)=g(x)
$$

where $F$ represents a general nonlinear ordinary differential operator involving both linear and nonlinear parts and $g(x)$ is a given function. The linear terms in $F y$ are decomposed into $L y+R y$, where $L$ is an invertible operator, which for AMDM is taken as the highest-order derivative, and $R$ is the remainder of the linear operator. Equation (1) can now be written as

$$
L y+R y+N y=g,
$$

where $N y$ represents the nonlinear terms of $F y$ and equation (2) represents an initial value or boundary value problem.

On solving for $L y$, equation (2) can be transformed to

$$
y=\Phi+L^{-1}(g)-L^{-1}(R y)-L^{-1}(N y),
$$

where $\Phi$ is the integration constant and $L \Phi=0$ is satisfied. To use the AMDM, $y$ is decomposed into the infinite sum of a convergent series:

$$
y=\sum_{m=0}^{\infty} C_{m} x^{m},
$$

and the nonlinear term is decomposed using Adomian polynomials, $A_{m}$ :

$$
N y=\sum_{m=0}^{\infty} x^{m} A_{m}\left(C_{0}, C_{1}, \ldots, C_{m}\right) .
$$

The function $g(x)$ can also be decomposed as

$$
g(x)=\sum_{m=0}^{\infty} g_{m} x^{m}
$$

Putting equations (4)-(6) into equation (3) gives

$$
\begin{aligned}
y= & \sum_{m=0}^{\infty} C_{m} x^{m}=\Phi+L^{-1}\left(\sum_{m=0}^{\infty} g_{m} x^{m}\right) \\
& -L^{-1} R\left(\sum_{m=0}^{\infty} C_{m} x^{m}\right) \\
& -L^{-1}\left(\sum_{m=0}^{\infty} x^{m} A_{m}\left(C_{0}, C_{1}, \ldots, C_{m}\right)\right) .
\end{aligned}
$$

The coefficient $C_{m}$ can be calculated using a recurrence relation and the power series solutions of linear homogeneous differential equations in initial value problems yield simple recurrence relations for the coefficient $C_{m}$. In practice, the coefficients cannot be determined exactly, and the solutions can only be approximated by a truncated series $\sum_{m=0}^{n-1} C_{m} x^{m}$.

\section{Mathematical Formulation}

In this work, a uniform beam under axial load while resting on a Winkler foundation is considered. As shown in Figure 1 , the beam has a length, $l$, a uniform rectangular cross section, $A$, a cross-sectional moment of inertia, $I$, and the beam is considered as made of isotropic material with a modulus of elasticity, $E$, and density, $\rho$.

The model for the foundation is the relatively simple Winkler model whose stiffness changes along the beam length and is a function of the spatial coordinate along the beam in the $x$ direction.

According to the theory of structural vibrations [26, 27], on using the Euler-Bernoulli beam model, the strain energy induced by a large displacement amplitude is given by 


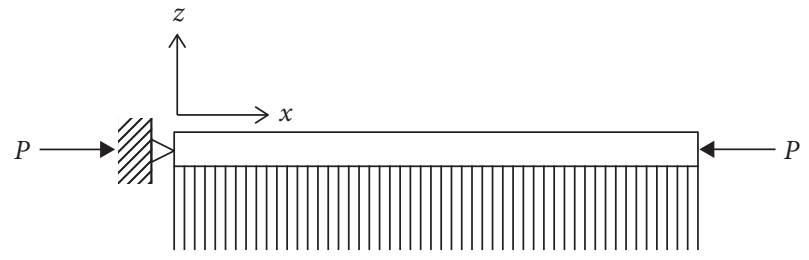

Figure 1: Beam under axial loading while resting on an elastic foundation.

$$
\begin{aligned}
U= & \frac{1}{2} \int_{0}^{l} E I\left(\frac{\partial^{2} w(x, t)}{\partial x^{2}}\right)^{2} d x \\
& +\frac{1}{2} \int_{0}^{l} E A\left(\frac{\partial u(x, t)}{\partial x}+\frac{1}{2}\left(\frac{\partial w(x, t)}{\partial x}\right)^{2}\right)^{2} d x \\
& +\frac{1}{2} \int_{0}^{l} k_{w}(x) w(x, t)^{2} d x
\end{aligned}
$$

The large amplitude of the vibrations necessitates the inclusion of the nonlinear term shown in equation (8).

Here, $u$ and $w$ are the axial and transverse displacements, respectively, and $k_{w}(x)$ is the foundation stiffness coefficient. The kinetic energy is given by

$$
T=\frac{1}{2} \int_{0}^{l} \rho A\left(\frac{\partial w(x, t)}{\partial t}\right)^{2} d x
$$

The external work done by the axial load is

$$
W=\frac{P}{2} \int_{0}^{l}\left(\frac{\partial w(x, t)}{\partial x}\right)^{2} d x .
$$

By invoking Hamilton's principle and using the Lagrangian of the system,

$$
\delta \int_{t_{1}}^{t_{2}}(T-U+W) d t=0 .
$$

On substituting equations (8)-(10) into equation (11), the following governing equation can be obtained after eliminating axial displacement:

$$
\begin{aligned}
& E I \frac{\partial^{4} w(x, t)}{\partial x^{4}}+\rho A \frac{\partial^{2} w(x, t)}{\partial t^{2}}+P \frac{\partial^{2} w(x, t)}{\partial x^{2}} \\
& \quad+k_{w}(x) w(x, t)-\frac{E A}{2 l} \frac{\partial^{2} w(x, t)}{\partial x^{2}} \int_{0}^{l}\left(\frac{\partial w(x, t)}{\partial x}\right)^{2} d x=0,
\end{aligned}
$$

where $k_{0}$ is a constant and $g(x)$ is a function of the spatial coordinate along the beam length.

According to modal analysis for harmonic-free vibration, $w(x, t)$ can be separable in space and time as

$$
w(x, t)=\phi(x) h(t)
$$

where $\phi(x)$ is the modal deflection and $h(t)$ is a harmonic function of time $t$. If $\omega$ denoted the circular frequency of $h(t)$, then $\partial^{2} w(x, t) / \partial t^{2}=-\omega^{2} \phi(x) h(t)$ and the eigenvalue problem of equation (12) reduces to

$$
\begin{aligned}
& E I \frac{d^{4} \phi(x)}{d x^{4}}+P \frac{d^{2} \phi(x)}{d x^{2}}-\frac{E A}{2 l} \frac{d^{2} \phi(x)}{d x^{2}} \\
& \int_{0}^{l}\left(\frac{d \phi(x)}{d x}\right)^{2} d x+k_{w} \phi(x)-\rho A \omega^{2} \phi(x)=0 .
\end{aligned}
$$

Equation (14) is now made nondimensional using

$$
\begin{gathered}
X=\frac{x}{l}, \\
\phi(X)=\frac{\phi(x)}{l}, \\
\bar{P}=\frac{P l^{2}}{E I}, \\
K_{0}=\frac{k_{w} l^{4}}{E I}, \\
\lambda=\frac{\rho A \omega^{2} l^{4}}{E I},
\end{gathered}
$$

and becomes

$$
\begin{aligned}
& \frac{d^{4} \phi(X)}{d X^{4}}+\bar{P} \frac{d^{2} \phi(X)}{d X^{2}}-\frac{1}{2} \frac{d^{2} \phi(X)}{d X^{2}} \\
& \quad \int_{0}^{1}\left(\frac{d \phi(X)}{d X}\right)^{2} d X+\left(K_{0}-\lambda\right) \phi(X)=0 .
\end{aligned}
$$

\section{Boundary Conditions}

Two cases are considered in this work, namely, beams which are clamped-clamped and clamped-free, respectively:

For the clamped-clamped case, the boundary conditions at $X=0$ and $X=1$ are

$$
\phi(X)=\frac{d \phi(X)}{d X}=0 .
$$

For the clamped-free case, the boundary conditions at $X=0$ and $X=1$ are

$$
\begin{aligned}
\phi(0) & =\frac{d \phi(0)}{d X}=0, \\
\frac{d^{2} \phi(1)}{d X^{2}} & =0 \\
\frac{d^{3} \phi(1)}{d X^{3}}+\bar{P} \frac{d \phi(1)}{d X} & =0
\end{aligned}
$$

It is convenient for the AMDM to describe boundary conditions in terms of rotational and translational flexible ends as shown in Figure 2.

The boundary conditions can be written in the dimensionless form as 


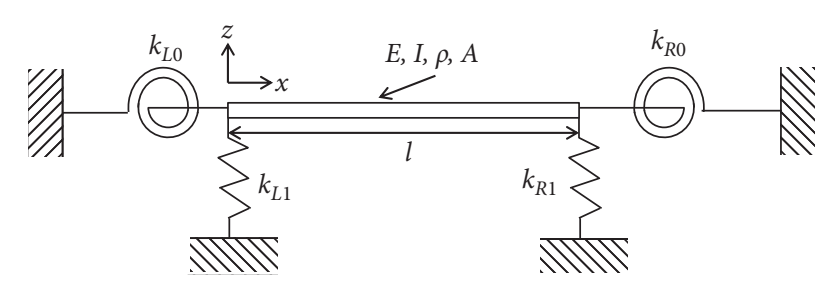

Figure 2: Boundary condition described by rotational and translational flexible ends.

$$
\begin{aligned}
\frac{d^{2} \phi(0)}{d X^{2}}-\kappa_{L 0} \frac{d \phi(0)}{d X} & =0, \\
\frac{d^{3} \phi(0)}{d X^{3}}+\kappa_{L 1} \phi(0) & =0, \\
\frac{d^{2} \phi(1)}{d X^{2}}+\kappa_{R 0} \frac{d \phi(1)}{d X} & =0, \\
\frac{d^{3} \phi(1)}{d X^{3}}+\bar{P} \frac{d \phi(1)}{d X}-\kappa_{R 1} \phi(1) & =0,
\end{aligned}
$$

where the coefficients are nondimensionalized as

$$
\begin{gathered}
\kappa_{L 1}=\frac{k_{L 1} l^{3}}{E I}, \\
\kappa_{R 1}=\frac{k_{R 1} l^{3}}{E I}, \\
\kappa_{L 0}=\frac{k_{L 0} l}{E I}, \\
\kappa_{R 0}=\frac{k_{R 0} l}{E I} .
\end{gathered}
$$

\section{Application of the Adomian Modified Decomposition Method (AMDM)}

According to the AMDM, $\phi(X)$ in equation (16) can be expressed as an infinite series, i.e.,

$$
\phi(X)=\sum_{m=0}^{\infty} C_{m} X^{m}
$$

where the unknown coefficients, $C_{m}$, are determined recurrently. If a linear operator $G \equiv d^{4} / d X^{4}$ is used, then the inverse operator of $G$ is a four fold operator defined as

$$
G^{-1}=\int_{0}^{x} \int_{0}^{x} \int_{0}^{x} \int_{0}^{x}(\cdots) d X d X d X d X .
$$

Equation (16) now can be written as

$$
\begin{aligned}
\phi(X)= & \Phi(X)-G^{-1}\left\{\bar{P} \frac{d^{2} \phi(X)}{d X^{2}}-\frac{1}{2} \frac{d^{2} \phi(X)}{d X^{2}}\right. \\
& \left.\cdot \int_{0}^{1}\left(\frac{d \phi(X)}{d X}\right)^{2} d X+\left(K_{0}-\lambda\right) \phi(X)\right\},
\end{aligned}
$$

where $\Phi(X)$ is a polynomial depending on the boundary conditions.

5.1. Nonlinear Fredholm Integral Coefficient. The terms in equation (23) are linear except for the nonlinear Fredholm integral coefficient shown in equation (24). Before continuing with the main solution method, the nonlinear term will be treated first through the use of appropriate Cauchy products. Consider

$$
\frac{1}{2}\left(\int_{0}^{1}\left(\frac{d \phi(X)}{d X}\right)^{2} d X\right) \frac{d^{2} \phi(X)}{d X^{2}} .
$$

Use is now made of the term

$$
\phi(X)=\sum_{m=0}^{\infty} C_{m}\left(X-X_{0}\right)^{m} \text {. }
$$

Differentiating

$$
\frac{d \phi(X)}{d X}=\sum_{m=0}^{\infty}(m+1) C_{m+1}\left(X-X_{0}\right)^{m}=\sum_{m=0}^{\infty} b_{m}\left(X-X_{0}\right)^{m},
$$

$\frac{d^{2} \phi(X)}{d X^{2}}=\sum_{m=0}^{\infty}(m+1)(m+2) C_{m+2}\left(X-X_{0}\right)^{m}$.

On setting $X_{0}=0$,

$$
\frac{d^{2} \phi(X)}{d X^{2}}=\sum_{m=0}^{\infty}(m+1)(m+2) C_{m+2}(X)^{m}=\sum_{m=0}^{\infty} b_{m} X^{m} .
$$

So

$$
\begin{aligned}
\left(\frac{d \phi(X)}{d X}\right)^{2} & =\left(\sum_{m=0}^{\infty} b_{m}\left(X-X_{0}\right)^{m}\right)\left(\sum_{l=0}^{\infty} b_{l}\left(X-X_{0}\right)^{l}\right) \\
& =\sum_{m=0}^{\infty} B_{m}\left(X-X_{0}\right)^{m},
\end{aligned}
$$

where $B_{m}=\sum_{l=0}^{m} b_{l} b_{m-l}$.

So

$$
\begin{aligned}
\frac{1}{2} \int_{0}^{1}\left(\frac{d \phi(X)}{d X}\right)^{2} d X & =\frac{1}{2} \int_{0}^{1} \sum_{m=0}^{\infty} B_{m}\left(X-X_{0}\right)^{m} d X \\
& =\frac{1}{2} \sum_{m=0}^{\infty} \int_{0}^{1} B_{m}\left(X-X_{0}\right)^{m} d X \\
& =\left[\frac{1}{2} \sum_{m=0}^{\infty} B_{m} \frac{\left(X-X_{0}\right)^{m+1}}{m+1}\right]_{X=0}^{X=1} .
\end{aligned}
$$

As $X_{0}=0$ in the current analysis, then

$$
\frac{1}{2} \int_{0}^{1}\left(\frac{d \phi(X)}{d X}\right)^{2} d X=\sum_{m=0}^{\infty} \frac{B_{m}}{2(m+1)}=\sum_{m=0}^{\infty} \alpha_{m} .
$$

Combining the two strands of analysis gives 


$$
\begin{aligned}
\frac{1}{2}\left(\int_{0}^{1}\left(\frac{d \phi(X)}{d X}\right)^{2} d X\right) \frac{d^{2} \phi(X)}{d X^{2}} & =\sum_{m=0}^{\infty} \alpha_{m} \sum_{l=0}^{\infty} \beta_{l} X^{m} \\
& =\sum_{m=0}^{\infty} X^{m} \sum_{l=0}^{m} \alpha_{l} \beta_{m-l} \\
& =\sum_{m=0}^{\infty} \zeta_{m} X^{m}
\end{aligned}
$$

where

$$
\zeta_{m}=\sum_{l=0}^{m} \alpha_{l} \beta_{m-l}
$$

5.2. Linear and Nonlinear Terms Combined. Using the analysis of the last subsection and $\phi(X)=\sum_{m=0}^{\infty} C_{m} X^{m}$, equation (23) now becomes

$$
\begin{aligned}
\phi(X)= & \Phi(X)+G^{-1}\left\{-\bar{P} \sum_{m=0}^{\infty}(m+1)(m+2) C_{m+2} X^{m}\right. \\
& \left.+\sum_{m=0}^{\infty} \zeta_{m} X^{m}-\left(K_{0}-\lambda\right) \sum_{m=0}^{\infty} C_{m} X^{m}\right\},
\end{aligned}
$$

where $\Phi(X)=\sum_{m=0}^{3} C_{m} X^{m}=\phi(0)+\phi^{\prime}(0) X+\phi^{\prime \prime}(0) X^{2} / 2+$ $\phi^{\prime \prime \prime}(0) X^{3} / 6$ is the initial term. The recurrence relation for the coefficients $C_{m}$ can now be stated as

$$
\begin{aligned}
& C_{0}=\phi(0), \\
& C_{1}=\phi^{\prime}(0), \\
& C_{2}=\frac{\phi^{\prime \prime}(0)}{2}, \\
& C_{3}=\frac{\phi^{\prime \prime \prime}(0)}{6},
\end{aligned}
$$

and for $m \geq 4$ as

$$
\begin{aligned}
C_{m}= & \frac{1}{m(m-1)(m-2)(m-3)} \\
& \sum_{j=0}^{m-4}\left[-\bar{P}(j+1)(j+2) C_{j+2}+\zeta_{j}-\left(K_{0}-\lambda\right) C_{j}\right] .
\end{aligned}
$$

The coefficients $C_{m}$ can be found from the recurrence equations (34) and (35), and the solution for $\phi(X)$ is calculated using equation (33). The series solution is $\phi(X)=\sum_{m=0}^{\infty} C_{m} X^{m}$, although all of the coefficients $C_{m}$ cannot be determined, and thus, the solutions must be approximated by the truncated series $\sum_{m=0}^{n-1} C_{m} X^{m}$ and successive approximations are $\phi^{[n]}(X)=\sum_{m=0}^{n-1} C_{m} X^{m}$, as $n$ increases and the boundary conditions are met.

Thus, $\quad \phi^{[1]}(X)=C_{0}, \quad \phi^{[2]}(X)=\phi^{[1]}(X)+C_{1} X$, $\phi^{[3]}(X)=\phi^{[2]}(X)+C_{2} X^{2}$, and $\phi^{[4]}(X)=\phi^{3}(X)+C_{3} X^{3}$ serve as approximate solutions with increasing accuracy as $n \longrightarrow \infty$. The four coefficients $C_{j}(j=0,1,2,3)$ depend on the boundary conditions used (either equation (17) or (18)). For example, for the clamped-free boundary conditions at $X=0$, the two coefficients $C_{0}$ and $C_{1}$ can be chosen as arbitrary constants, and the other two coefficients $C_{2}$ and $C_{3}$ can be expressed as functions of $C_{0}$ and $C_{1}$. Thus, from equations (19) and (34), the following is obtained:

$$
\begin{aligned}
& C_{2}=\frac{\kappa_{L 0}}{2} C_{1}, \\
& C_{3}=-\frac{\kappa_{R 0}}{6} C_{0} .
\end{aligned}
$$

Thus the initial term $\Phi(X)$ is only a function of $C_{0}$ and $C_{1}$, and from the recurrence relation of equation (36), the coefficients $C_{m}(m \geq 4)$ are functions of $C_{0}, C_{1}$, and $\lambda$. By substituting $\phi^{[n]}(X)$ into the boundary conditions of equation (19) when $X=1$, we have

$$
f_{r 0}^{[n]}(\lambda) C_{0}+f_{r 1}^{[n]}(\lambda) C_{1}=0, \quad r=1,2 .
$$

For nontrivial solutions of $C_{0}$ and $C_{1}$, the frequency equation is given as

$$
\left|\begin{array}{ll}
f_{10}^{[n]}(\lambda) & f_{11}^{[n]}(\lambda) \\
f_{20}^{[n]}(\lambda) & f_{21}^{[n]}(\lambda)
\end{array}\right|=0 .
$$

The $i^{\text {th }}$ estimated eigenvalue $\lambda_{(i)}^{[n]}$ corresponding to $m$ is obtained from equation (38), i.e., the $i^{\text {th }}$ estimated dimensionless natural frequency $\Omega_{n(i)}^{[n]}=\sqrt{\lambda_{(i)}^{[n]}}$ is also obtained and $n$ is determined by

$$
\left|\Omega_{n(i)}^{[n]}-\Omega_{n(i)}^{[n-1]}\right| \leq \varepsilon
$$

where $\Omega_{n(i)}^{[n-1]}$ is the $i^{\text {th }}$ estimated dimensionless natural frequency corresponding to $n-1$ and $\varepsilon$ is a preset sufficiently small value. If equation (39) is satisfied, then $\Omega_{n(i)}^{[n]}$ is the $i^{\text {th }}$ dimensionless natural frequency $\Omega_{n(i)}$. By substituting $\Omega_{n(i)}^{[n]}$ into equation (37),

$$
C_{1}=-\frac{f_{r 0}^{[n]}\left(\Omega_{n(i)}^{[n]}\right)}{f_{r 1}^{[n]}\left(\Omega_{n(i)}^{[n]}\right)} C_{0}, \quad r=1,2,
$$

and all of the other coefficients $C_{m}$ can be obtained from equations (34) and (35). Furthermore, the $i^{\text {th }}$ mode shape $\phi_{i}^{[n]}$ corresponding to the $i^{\text {th }}$ eigenvalue $\Omega_{n(i)}^{[n]}$ is obtained by

$$
\phi_{i}^{[n]}(X)=\sum_{m=0}^{n-1} C_{m}^{[i]} X^{m},
$$

where $C_{m}^{[i]}(X)$ is $C_{m}(X)$ in which $\lambda$ is substituted by $\lambda_{i}$ and $\phi_{i}^{[n]}$ is the $i^{\text {th }}$ eigenfunction corresponding to the $i^{\text {th }}$ eigenvalue $\lambda_{i}$. By normalizing equation (41), the $i$ th normalised eigenfunction is defined as

$$
\bar{\phi}_{i}^{[n]}(X)=\frac{\phi_{i}^{[n]}(X)}{\sqrt{\int_{0}^{1}\left[\phi_{i}^{[n]}(X)\right]^{2} d X}},
$$


where $\bar{\phi}_{i}^{[n]}(X)$ is the $i^{\text {th }}$ mode shape function of the beam corresponding to the $i^{\text {th }}$ natural frequency $\omega_{i}^{[n]}=\sqrt{\lambda_{i}^{[n]} \sqrt{E I / \rho A l^{4}}}=\Omega_{n(i)}^{[n]} \sqrt{E I / \rho A l^{4}}$.

This general theory is now applied to a uniform Euler-Bernoulli beam under different boundary conditions.

\section{Numerical Results}

6.1. Clamped-Free Uniform Beam. The first case considered is the clamped-free uniform beam resting on an elastic foundation and experiencing axial compressive force The case was chosen to test (validate) the accuracy of the AMDM.

The boundary conditions are as given in equations (18) and (19) with the spring constants becoming $\kappa_{L 0} \longrightarrow$ $\infty, \kappa_{R 0} \longrightarrow 0, \kappa_{L 1}=\infty, \kappa_{R 1}=0$.

When $X=0$, the first two boundary conditions of equation (19) yield the relationships shown in equation (36), and when $X=1$ by substituting $\phi^{[n]}(X)=\sum_{m=0}^{n-1} C_{m} X^{m}$ into the last two boundary conditions of equation (19), the following two algebraic equations (written in full) involving $C_{0}$ and $C_{1}$ are obtained:

$$
\begin{aligned}
& \sum_{m=0}^{n-3}(m+1)(m+2) C_{m+2}+\kappa_{R 0} \sum_{m=0}^{n-2}(m+1) C_{m+1} \\
& \quad=f_{11}^{[n]}(\lambda) C_{0}+f_{12}^{[n]}(\lambda) C_{1}=0 \\
& \sum_{m=0}^{n-4}(m+1)(m+2)(m+3) C_{m+3}+\bar{P} \sum_{m=0}^{n-2}(m+1) C_{m+1} \\
& \quad-\kappa_{R 1} \sum_{m=0}^{n-1} C_{m}=f_{21}^{[n]}(\lambda) C_{2}+f_{22}^{[n]}(\lambda) C_{3}=0 .
\end{aligned}
$$

The case of the clamped-free uniform beam without an elastic foundation support or without any axial force and with small vibration amplitude was first chosen to test (validate) the accuracy of the AMDM as comparisons can be made with what is already given in the literature. For this, the values of $\bar{P}, K_{0}$, and nonlinear term in equation (16) were set to zero. The first five natural frequencies, $\left(\Omega_{n(i)}, i=1, \ldots, 5\right)$ are shown in Table 1 and compared with those obtained by Reference [9]. Excellent agreement was found.

The results are shown in Table 2, with, and without, the nonlinear term given in equation (16). Here, $\Omega_{\text {non (i) }}$ are the results with the nonlinear term used and $\Omega_{n(i)}$ are the results when the nonlinear term was not used.

It can be seen from Table 2 that the results agree with those of Reference [27] fairly well where the nonlinear term is absent although generally the present results are slightly higher than those calculated by Chen [27]. Also shown in Table 2 are the results calculated for large amplitudes. This was effected through the inclusion of the nonlinear term of equation (16). The ratio of results calculated with and without the inclusion of the nonlinear term is also given in Table 2. The presence of the nonlinear terms increases quite substantially the natural frequencies, and it can be seen that this increase grows in line with increasing the mode number.
Figure 3 presents the variation of natural frequency modes with increase in axial force and the foundation stiffness. During the variation of the axial force the elastic stiffness, $K_{0}$, was held constant and for the variation of the elastic stiffness, the axial force, $\bar{P}$, was held constant. It is noticeable that the first mode is significantly affected by both variations in comparison with the higher modes, and in particular, the first mode natural frequency is greatly affected by increasing in axial force.

By substituting the converged $\Omega_{n(1)}^{[n]}$ into equations (21) and (35) and normalizing the result using equation (42), a polynomial can be obtained to describe the first mode shape function. The same procedure can be employed for other natural frequencies to find the mode shapes for higher mode numbers. The variation of the first and third mode shapes for various Winkler parameters is illustrated on Figure 4. Here, the axial force was set to zero. The changes in the mode shape were not too significant until the value of $K_{0}$ became reasonably large. It can be seen from the results for the third mode that the increase in elastic stiffness affects both the amplitude and the phase of the shape function.

A similar exercise was carried out to ascertain the trends for the mode shapes when the axial force was increased or when the beam was tensioned as opposed to being compressed. The effects on the first and third modes are shown in Figure 5. Here, the elastic stiffness was held constant at one. It can be seen that the greatest effect was on the first mode and, except at the extrema, increasing the axial load had very little effect on higher modes.

Important to this study was the speed of obtaining accurate converged solutions. An example of the rate of convergence is given in Figure 6 for the case of clamped-free boundary conditions with $\bar{P}=0$ and $K_{0}=1$. As can be seen, the AMDM method was fast converging with results for the first mode obtained after a very few iterations. Convergence for higher modes took longer, although not prohibitively.

6.2. Clamped-Clamped Uniform Beam. For this case, the boundary conditions are as given in equations (17) and (19) with the spring constants becoming $\kappa_{L 0} \longrightarrow \infty$, $\kappa_{R 0} \longrightarrow \infty, \kappa_{L 1} \longrightarrow \infty$, and $\kappa_{R 1} \longrightarrow \infty:$

$$
\begin{aligned}
C_{2} & =\frac{\kappa_{L 0}}{2} C_{1}, \\
C_{3} & =-\frac{\kappa_{L 1}}{6} C_{0}, \\
\sum_{m=0}^{n-1} C_{m} & =f_{11}^{[n]}(\lambda) C_{0}+f_{12}^{[n]}(\lambda) C_{1}=0, \\
\sum_{m=0}^{n-2}(m+1) C_{m+1} & =f_{21}^{[n]}(\lambda) C_{0}+f_{22}^{[n]}(\lambda) C_{1}=0 .
\end{aligned}
$$

Calculations were made for the clamped-clamped beam of the natural frequencies with no axial force and for two values of foundation stiffness as shown in Table 3 . The results for the present method compare well with those reported in 
TABLE 1: First five natural frequencies $\left(\Omega_{n(i)}, i=1, \ldots, 5\right)$ for the small vibration amplitude.

\begin{tabular}{lccccc}
\hline Method & $\Omega_{n(1)}$ & $\Omega_{n(2)}$ & $\Omega_{n(3)}$ & $\Omega_{n(4)}$ & $\Omega_{n(5)}$ \\
\hline Present & 3.516010 & 22.034484 & 61.697213 & 120.901920 & 199.859536 \\
Reference [9] & 3.5160 & 22.0345 & 61.6972 & 120.902 & 199.860 \\
\hline
\end{tabular}

TABLE 2: Natural frequencies of the cantilever beam on an elastic foundation with $\bar{P}=0$ and $K_{0}=1$.

\begin{tabular}{lcccc}
\hline Mode $(i)$ & Reference [27] $\Omega_{n(i)}$ & Present $\Omega_{n(i)}$ & Present $\Omega_{\text {non }(i)}$ & Present $\Omega_{\text {non }(i)} / \Omega_{n(i)}$ \\
\hline 1 & 3.66 & 3.6712 & 3.9869 & 1.089 \\
2 & 22.22 & 22.2343 & 24.4049 & 1.098 \\
3 & 62.69 & 62.7421 & 69.1014 & 1.101 \\
4 & 124.29 & 124.3811 & 138.0632 & 1.111 \\
\hline
\end{tabular}

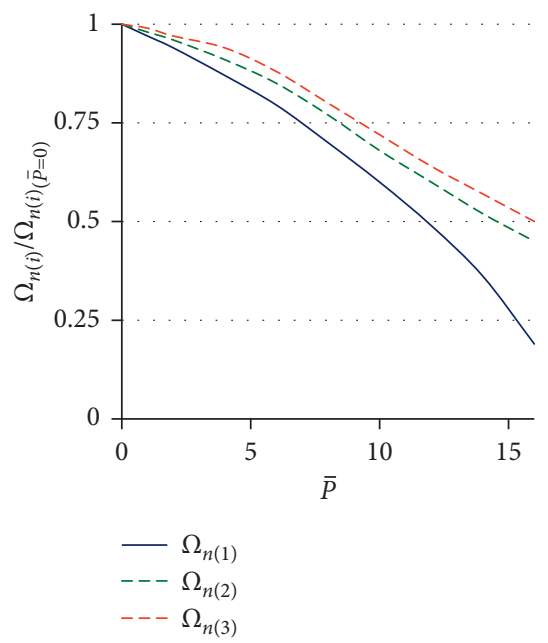

(a)

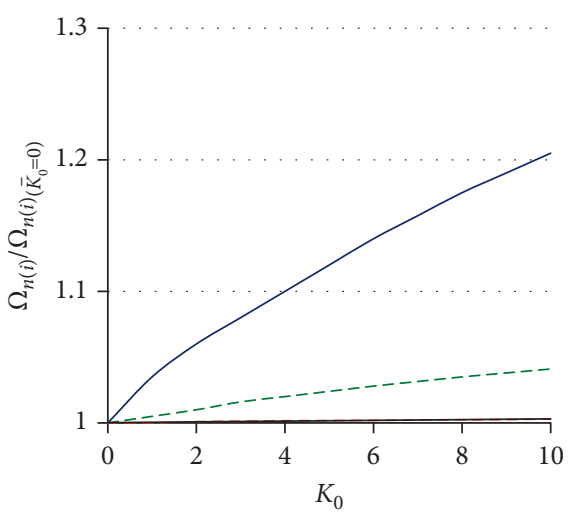

$$
\begin{aligned}
& -\Omega_{n(1)} \\
& ---\Omega_{n(2)} \\
& ---\Omega_{n(3)}
\end{aligned}
$$

(b)

FiguRE 3: Variation of frequency modes with axial force and foundation stiffness.

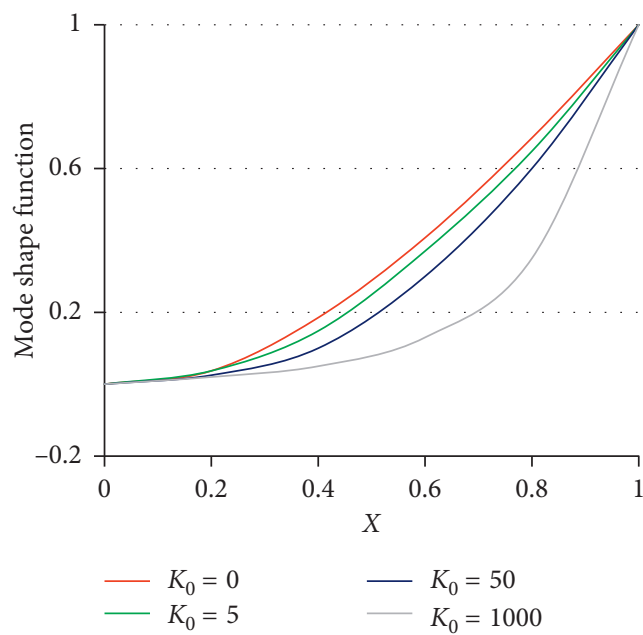

(a)

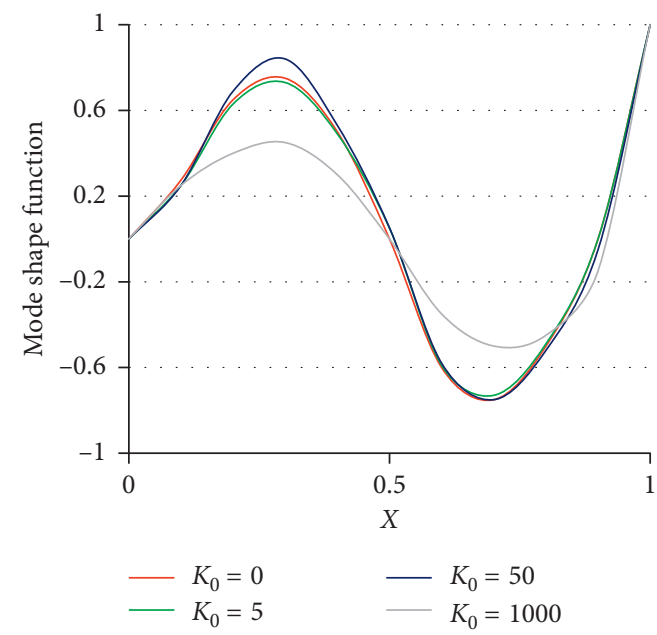

(b)

Figure 4: (a) First and (b) third mode shapes for various stiffness parameters with $\bar{P}=0$. 


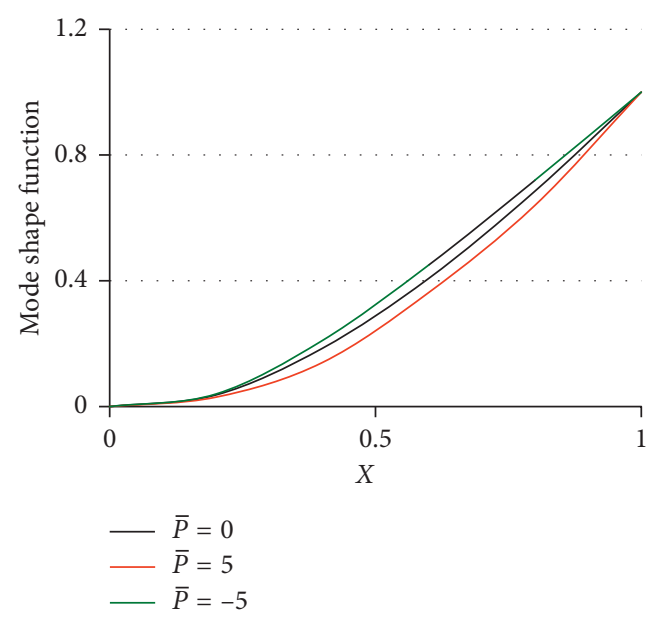

(a)

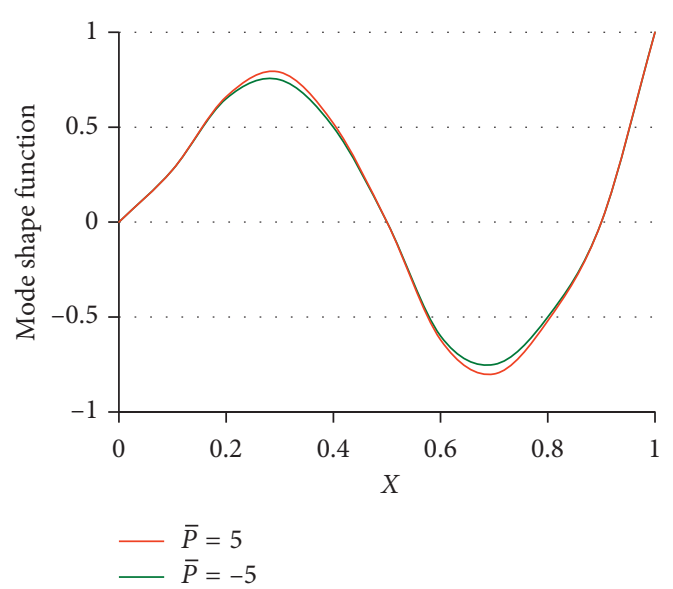

(b)

Figure 5: Effect of axial load on mode shapes with $K_{0}=1$. (a) $1^{\text {st }}$ mode. (b) $3^{\text {rd }}$ mode.

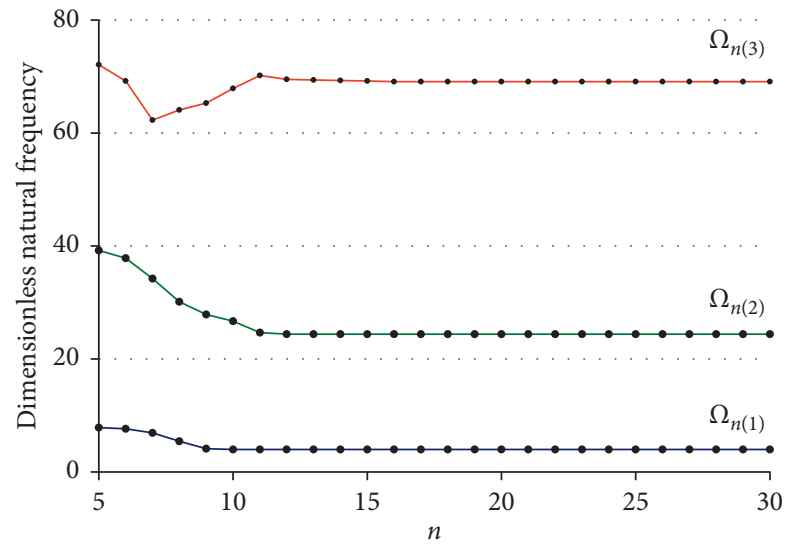

FIgURE 6: Convergence plots for natural frequencies when $\bar{P}=0$ and $K_{0}=1$.

TABLE 3: Natural frequencies of the clamped-clamped beam on an elastic foundation with $\bar{P}=0$ and without the nonlinear term.

\begin{tabular}{lcccccc}
\hline & & $K_{0}=0$ & & \multicolumn{2}{c}{$K_{0}=100$} \\
& Present & Reference [28] & Reference [29] & Present & Reference [28] & Reference [29] \\
\hline$\Omega_{n(1)}$ & 4.73004 & 4.7314 & 4.73 & 4.95246 & 4.9515 & 7.95 \\
$\Omega_{n(2)}$ & 7.85320 & 7.8533 & 7.854 & 7.91103 & 7.9044 & 7.904 \\
$\Omega_{n(3)}$ & 10.9955 & 10.9908 & 10.996 & 11.0121 & 11.0096 & 11.014 \\
\hline
\end{tabular}

the literature. These results were obtained with the nonlinear term of equation (16) not used.

Table 4 shows results for the first four modes with and without the nonlinear term present. Again $\Omega_{\text {non (i) }}$ is the result when the nonlinear term is used, and $\Omega_{n(i)}$ is the result when the nonlinear term is not used. It can be seen that the present calculated values of $\Omega_{n(i)}$ are in good agreement although again most of the results are slightly higher than those calculated by Chen [27]. When the nonlinear term is included, the results for the natural frequencies increase with the ratio of increase found to be similar in magnitude to those reported by Mei [30].

Again polynomials were obtained to describe the first three mode shape functions for the clamped-clamped beam. The first three mode shapes for the clamped-clamped beam are shown in Figure 7 after normalization and compared well with those reported in the literature.

Finally, the present method of calculation was validated using a backbone curve for the clamped-clamped beam as shown in Figure 8. The first mode results reported by Gupta 
TABLE 4: Natural frequencies of the clamped-clamped beam on an elastic foundation with $\bar{P}=0$ and $K_{0}=1$.

\begin{tabular}{lcccc}
\hline $\begin{array}{l}\text { Mode } \\
(i)\end{array}$ & $\begin{array}{c}\text { Reference [27] } \\
\Omega_{n(i)}\end{array}$ & $\begin{array}{c}\text { Present } \\
\Omega_{n(i)}\end{array}$ & $\begin{array}{c}\text { Present } \\
\Omega_{\text {non }(i)}\end{array}$ & $\begin{array}{c}\text { Present } \\
\Omega_{\text {non }(i)} / \Omega_{n(i)}\end{array}$ \\
\hline 1 & 22.435 & 22.614 & 23.971 & 1.06 \\
2 & 62.094 & 62.051 & 74.461 & 1.19 \\
3 & 122.659 & 123.54 & 149.772 & 1.21 \\
4 & 204.935 & 205.332 & 256.665 & 1.25 \\
\hline
\end{tabular}

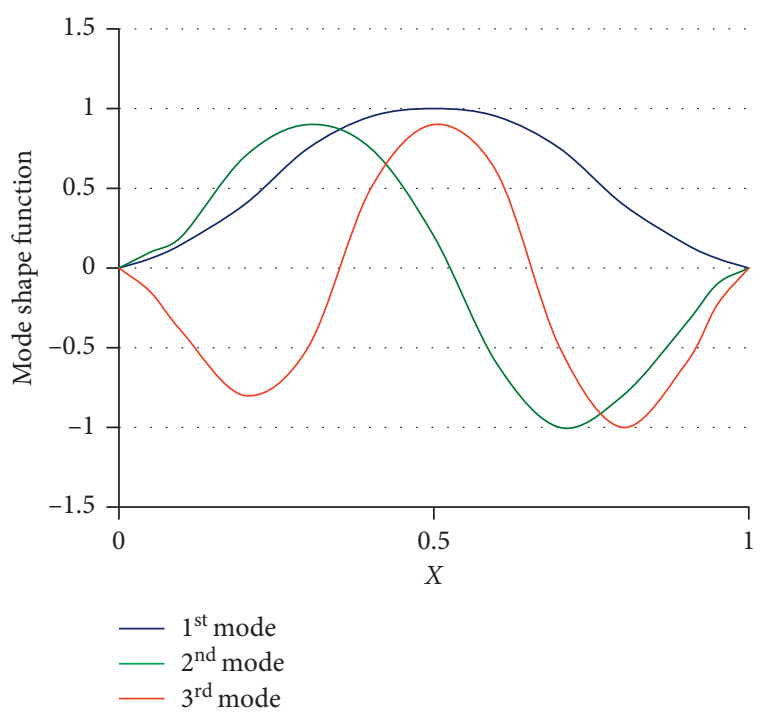

FIgURE 7: First three mode shapes for a clamped-clamped beam with $K_{0}=1$ and $\bar{P}=1$.

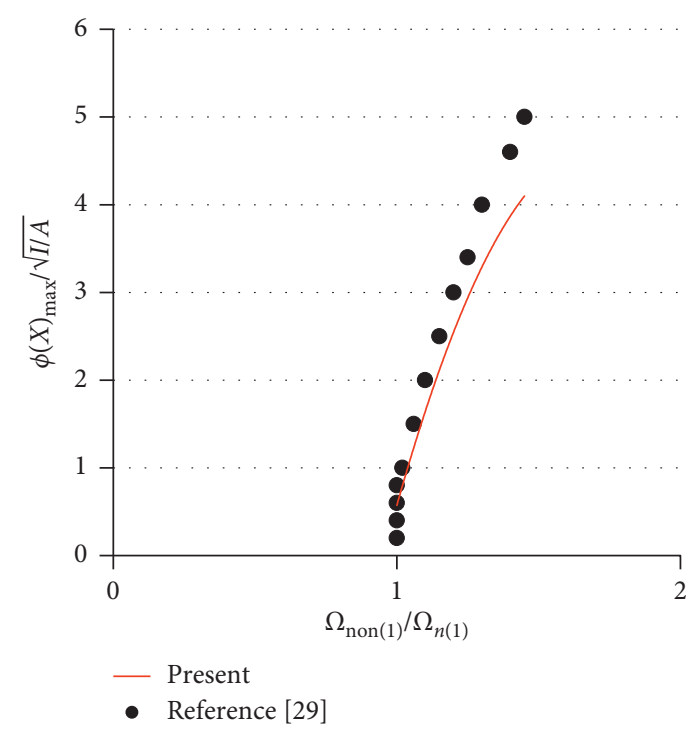

Figure 8: Comparison of backbone curves for the $1^{\text {st }}$ mode of the clamped-clamped beam.

et al. [31] were chosen with reasonable agreement found with a similar trend found between the two result sets, as illustrated in Figure 8. Here, the value of the foundation stiffness was set at zero.

\section{Conclusions}

A fast, efficient, and accurate method of solution, namely, the Adomian modified decomposition method (AMDM) was developed to calculate natural vibrations of an Euler-Bernoulli beam with large amplitude resting on a Winkler foundation. The method is free of linearization, discretization, perturbation, or a priori assumptions and nonlinear terms are relatively easily treated. A practical advantage of the AMDM is the ease of applying the boundary conditions where the vibrational analysis for different boundary conditions simply involves changing the values of the corresponding parameters with no need to change the solution procedures or the algorithms employed.

The numerical comparisons for both boundary conditions used here indicate that the current numerical results are in satisfactory agreement with those found by other methods, with perhaps some of the results found here better than some reported.

\section{Data Availability}

The data used to support the findings of this study are available from the corresponding author upon request.

\section{Conflicts of Interest}

The authors declare that there are no conflicts of interest with respect to this paper.

\section{Acknowledgments}

This investigation was supported by Nazarbayev University Small Competitive Grant no. 090118FD5317 and ORAU Grant no. SOE2017003.

\section{References}

[1] J.-H. He, "Variational iteration method for autonomous ordinary differential systems," Applied Mathematics and Computation, vol. 114, no. 2-3, pp. 115-123, 2000.

[2] H. Tari, D. D. Ganji, and H. Babazadeh, "The application of He's variational iteration method to nonlinear equations arising in heat transfer," Physics Letters A, vol. 363, no. 3, pp. 213-217, 2007.

[3] M. Dehghan and F. Shakeri, "Application of He's variational iteration method for solving the Cauchy reaction-diffusion problem," Journal of Computational and Applied Mathematics, vol. 214, no. 2, pp. 435-446, 2008.

[4] Y. Khan and Q. Wu, "Homotopy perturbation transform method for nonlinear equations using He's polynomials," Computers \& Mathematics with Applications, vol. 61, no. 8, pp. 1963-1967, 2011.

[5] Y. Khan and M. Usman, "Modified homotopy perturbation transform method: a paradigm for nonlinear boundary layer problems," International Journal of Nonlinear Sciences and Numerical Simulation, vol. 15, no. 1, pp. 19-25, 2014.

[6] N. M. Auciello and M. J. Maurizi, "On the natural vibrations of tapered beams with attached inertia elements," Journal of Sound and Vibration, vol. 199, no. 3, pp. 522-530, 1997. 
[7] I. Elishakoff and R. Becquet, "Closed-form solutions for natural frequency for inhomogeneous beams with one sliding support and the other pinned," Journal of Sound and Vibration, vol. 238, no. 3, pp. 529-539, 2000.

[8] S. Kukla and I. Zamajska, "Application of Green's function method in free vibration analysis of non-uniform beams," Scientific Research of the Institute of Mathematics and Computer Science, vol. 1, no. 4, pp. 87-94, 2005.

[9] G. Wang and N. M. Wereley, "Free vibration analysis of rotating blades with uniform tapers," AIAA Journal, vol. 42, pp. 2429-2437, 2004.

[10] J. R. Banerjee, H. Su, and D. R. Jackson, "Free vibration of rotating tapered beams using the dynamic stiffness method," Journal of Sound and Vibration, vol. 298, no. 4-5, pp. 10341054, 2006.

[11] F. J. Shaker, "Effect of axial load on mode shapes and frequencies of beams," NASA TN D-8109, NASA, Washington, DC, USA, 1975.

[12] A. Bokaian, "Natural frequencies of beams under compressive axial loads," Journal of Sound and Vibration, vol. 126, no. 1, pp. 49-65, 1988.

[13] S. Kukla and B. Skalmierski, "The effect of axial loads on transverse vibrations of an Euler-Bernoulli beam," Journal of Theoretical and Applied Mechanics, vol. 31, no. 2, pp. 413-430, 1993.

[14] G. R. Bhashyam and G. Prathap, "Galerkin finite element method for non-linear beam vibrations," Journal of Sound and Vibration, vol. 72, pp. 191-203, 1980.

[15] E. Özkaya, M. Pakdemirli, and H. R. Öz, "Non-linear vibrations of a beam-mass system under different boundary conditions," Journal of Sound and Vibration, vol. 199, pp. 679-696, 1997.

[16] K. N. Saha, R. C. Kar, and P. K. Datta, "Dynamic stability of a rectangular plate on non-homogeneous winkler foundation," Computers \& Structures, vol. 63, no. 6, pp. 1213-1222, 1997.

[17] H. P. Lee, "Dynamic response of a Timoshenko beam on a Winkler foundation subjected to a moving mass," Applied Acoustics, vol. 55, no. 3, pp. 203-215, 1998.

[18] P. Ruge and C. Birk, “A comparison of infinite Timoshenko and Euler-Bernoulli beam models on Winkler foundation in the frequency- and time-domain," Journal of Sound and Vibration, vol. 304, no. 3-5, pp. 932-947, 2007.

[19] D. Zhou, "A general solution to vibrations of beams on variable Winkler elastic foundation," Computers \& Structures, vol. 47, no. 1, pp. 83-90, 1993.

[20] M. Eisenberger and J. Clastornik, "Vibrations and buckling of a beam on a variable Winkler elastic foundation," Journal of Sound and Vibration, vol. 115, no. 2, pp. 233-241, 1987.

[21] G. Adomian, Solving Frontier Problems of Physics: The Decomposition Method, Kluwer Academic Publishers, Boston, MA, USA, 1994.

[22] A.-M. Wazwaz, "Analytic treatment for variable coefficient fourth-order parabolic partial differential equations," Applied Mathematics and Computation, vol. 123, no. 2, pp. 219-227, 2001.

[23] D. Adair and M. Jaeger, "Simulation of tapered rotating beams with centrifugal stiffening using the Adomian decomposition method," Applied Mathematical Modelling, vol. 40, no. 4, pp. 3230-3241, 2016.

[24] Q. Mao, "Application of Adomain modified method for free vibration analysis of rotating beams," Mathematical Problems in Engineering, vol. 2013, Article ID 284720, 10 pages, 2013.
[25] S. Das, "A numerical solution of the vibration equation using modified decomposition method," Journal of Sound and Vibration, vol. 320, no. 3, pp. 576-583, 2009.

[26] S. Timoshenko, D. H. Young, and W. Weaver Jr., Vibration Problems in Engineering, John Wiley, New York, NY, USA, 1974.

[27] C.-N. Chen, "Vibration of prismatic beam on an elastic foundation by the differential quadrature element method," Computers \& Structures, vol. 77, no. 1, pp. 1-9, 2000.

[28] W. Q. Chen, C. F. Lü, and Z. G. Bian, "A mixed method for bending and free vibration of beams resting on a Pasternak elastic foundation," Applied Mathematical Modelling, vol. 28, no. 10 , pp. 877-890, 2004.

[29] M. A. De Rosa and M. J. Maurizi, "The influence of concentrated masses and Pasternak soil on the free vibrations of euler beams-exact solution," Journal of Sound and Vibration, vol. 212, no. 4, pp. 573-581, 1998.

[30] C. Mei, "Nonlinear vibration of beams by matrix displacement method," AIAA Journal, vol. 10, no. 3, pp. 355-357, 1972.

[31] R. K. Gupta, G. Jagadish Babu, G. Ranga Janardhan, and G. Venkateswara Rao, "Relatively simple finite element formulation for the large amplitude free vibrations of uniform beams," Finite Elements in Analysis and Design, vol. 45, no. 10, pp. 624-631, 2009. 


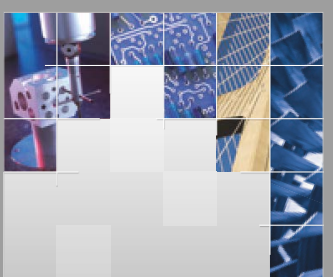

\section{Enfincering}
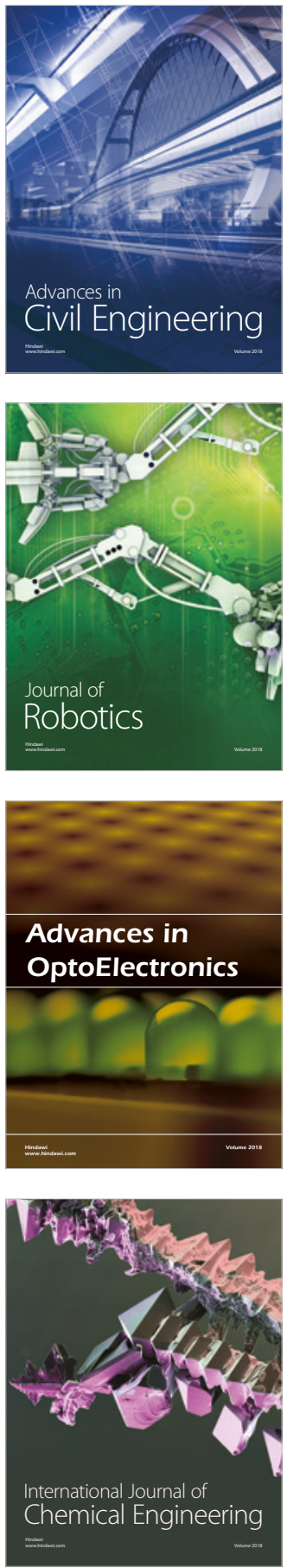

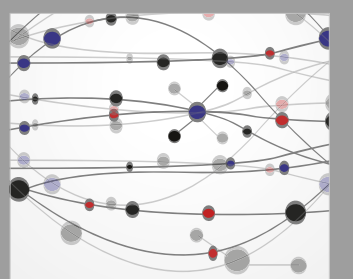

\section{Rotating \\ Machinery}

The Scientific World Journal

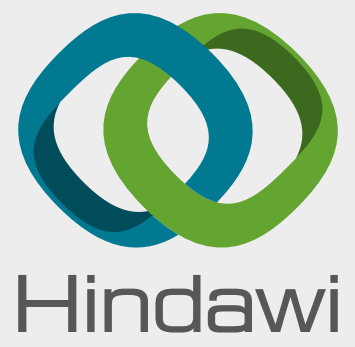

Submit your manuscripts at

www.hindawi.com
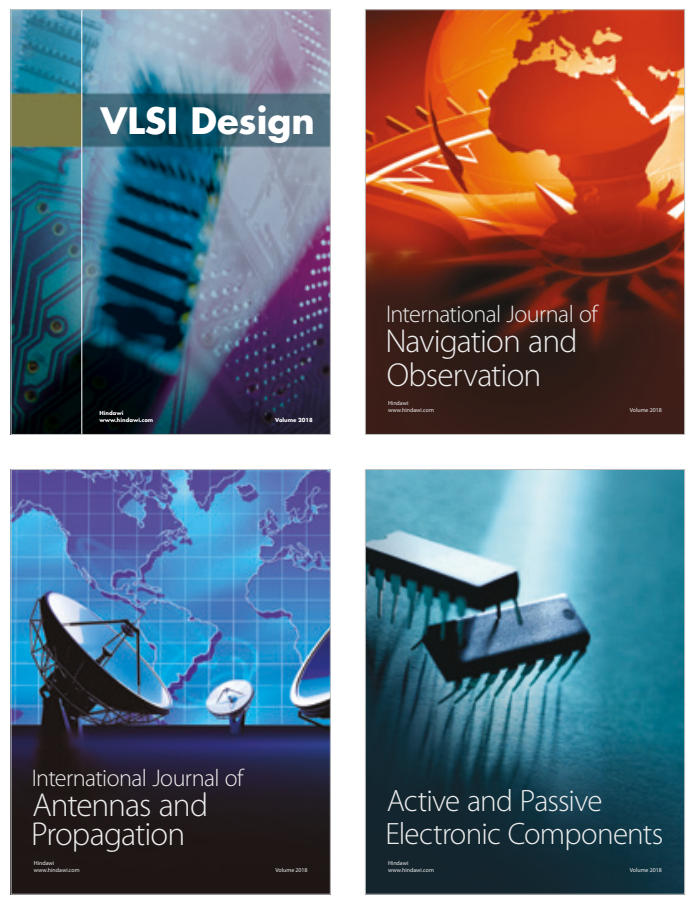
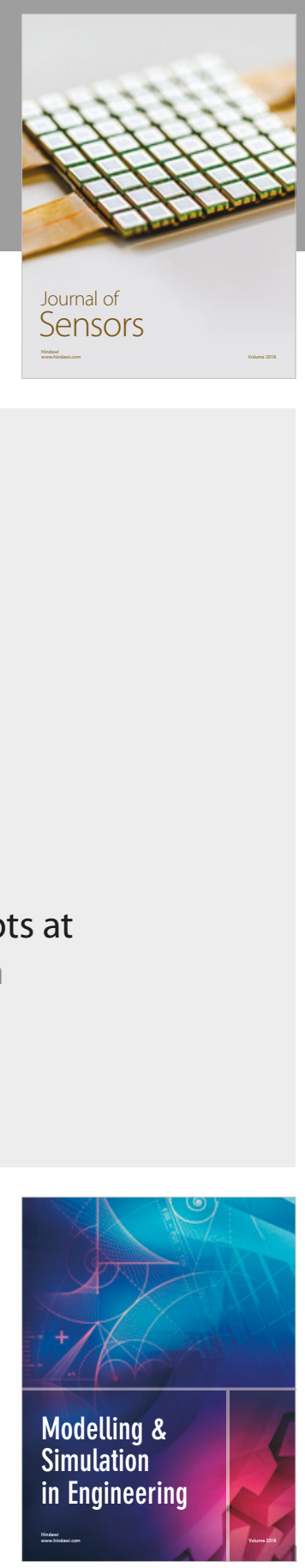

\section{Advances \\ Multimedia}
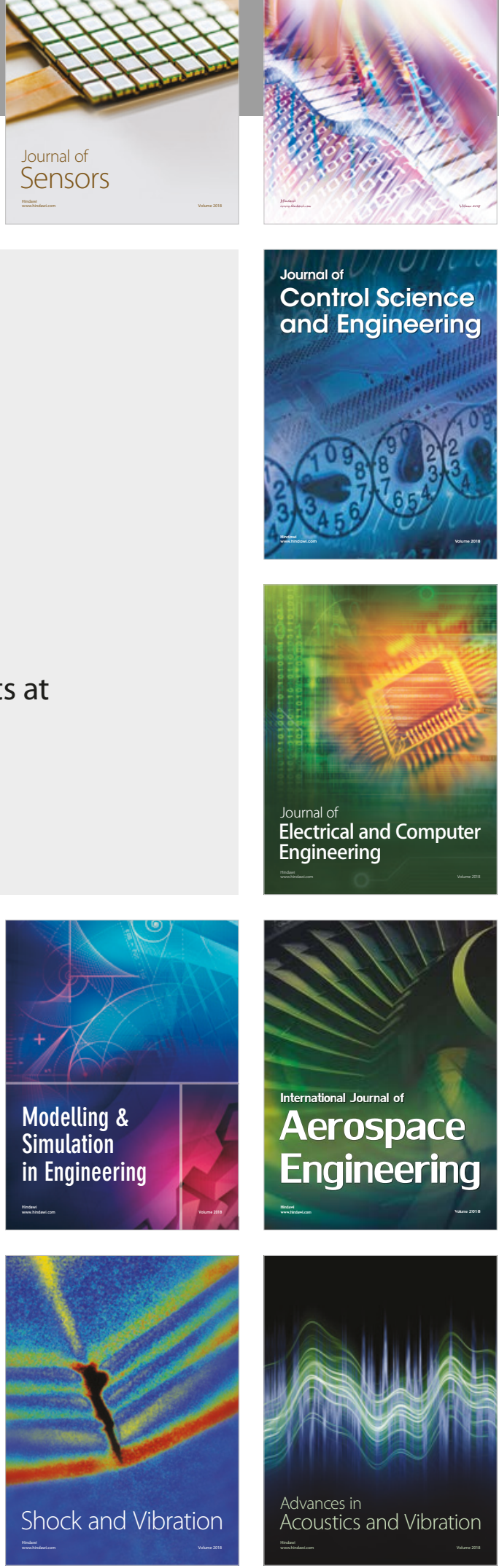